\title{
Mucopolysaccharidosis Type II and the G374sp Mutation
}

\author{
E. Martínez-Quintana F. Rodríguez-González \\ Complejo Hospitalario Universitario Insular-Materno Infantil, Las Palmas de Gran Canaria, Spain
}

\author{
Key Words \\ G374sp · Hunter - Mucopolysaccharidosis type II - Mutation · \\ Sleep apnea $\cdot$ Valve
}

\begin{abstract}
Mucopolysaccharidosis type II (MPS II), also known as Hunter syndrome, is a rare, $\mathrm{X}$-linked disease caused by a deficiency of the lysosomal enzyme iduronate-2-sulfatase, which catalyses a step in the catabolism of glycosaminoglycans resulting in accumulation of heparan and dermatan sulfate in many organs and tissues. This accumulation favors the appearance of neurologic involvement, severe airway obstruction, skeletal deformities, and cardiomyopathy, especially mitral and aortic valve regurgitation. In severe cases, obstructive airway disease and cardiac failure due to valvular dysfunction are the most common causes of death within the second decade of life. However, in mild cases, intelligence remains normal, stature is almost normal and death usually occurs due to cardiac failure in the fourth decade of life. We report the presentation, diagnosis, management, and outcome of 2 siblings with MPS II and the G374sp mutation at the nucleotide c.1246 of the gene encoding for the iduronate-2-sulfatase.

Copyright $\odot 2013$ S. Karger AG, Basel
\end{abstract}

Congenital metabolic disorders result from the absence or abnormality of an enzyme or its cofactor, leading to either accumulation or deficiency of a specific metabolite. In the case of mucopolysaccharidosis type II (MPS II) (Hunter syndrome, OMIM 309900), there is a deficiency of the lysosomal enzyme iduronate-2-sulfatase (IDS) which results in accumulation of the glycosaminoglycans (GAGs) heparan and dermatan sulfate in many organs and tissues favoring multisystem organ failure.

MPS II, with an incidence estimated between 1:110,000 and 132,500 live births, is a chronic and progressive disease classified as mild or severe according to the absence or presence of mental retardation and early behavioral disturbances [Young et al., 1982; Beck, 2011]. The clinical signs are similar to those found in the Hurler syndrome, although no corneal clouding is observed and dysostosis is generally less prominent. Upper respiratory tract infections, inguinal and umbilical hernia and joint stiffness represent the early symptoms and dysmorphism, dysostosis and kyphoscoliosis progress rapidly with age resulting in short stature [Froissart et al., 2007]. Obstructive airway disease and cardiac failure due to valvular dysfunction are the most common causes of death occurring as early as at the age of 15 , while the storage of GAGs in the brain can lead to delayed development with subsequent mental retardation. However, in mild cases, intelligence remains normal, stature is almost normal and death usually occurs due to cardiac failure in the fourth decade of life.

\section{KARGER}

E-Mail karger@karger.com

www.karger.com/msy (c) 2013 S. Karger AG, Basel

$1661-8769 / 13 / 0044-0203 \$ 38.00 / 0$
Dr. Efrén Martínez-Quintana

Servicio de Cardiología, Complejo Hospitalario Universitario Insular-Materno Infantil Avenida Marítima del Sur s/n ES-35016 Las Palmas de Gran Canaria (Spain)

E-Mail efrencardio@gmail.com 


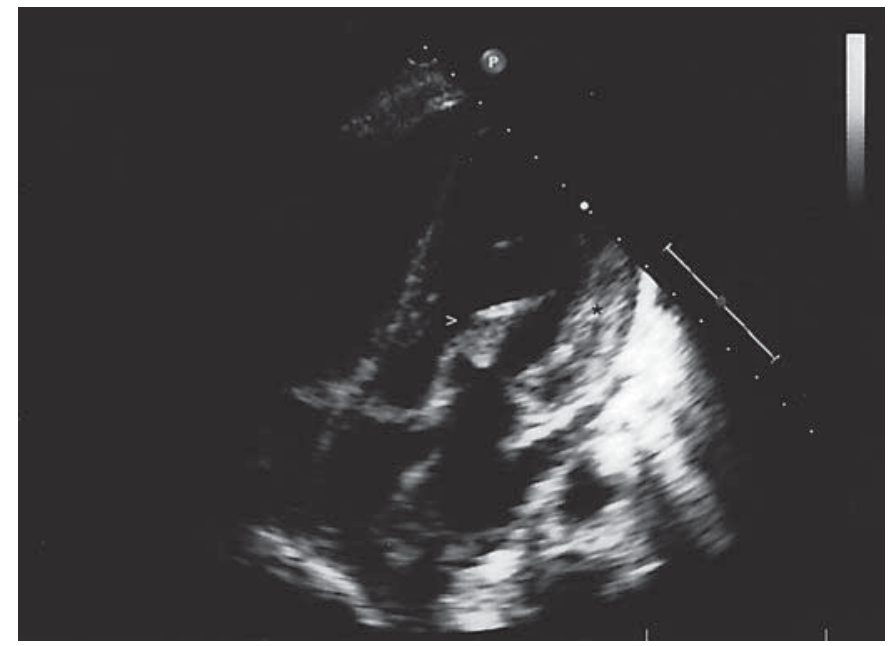

Fig. 1. Subcostal echocardiographic view showing anterior (arrowhead) and posterior mitral valve thickening with subvalvular involvement (asterisk). This thickening favors retraction, leaflet coaptation defect and valvular regurgitation.

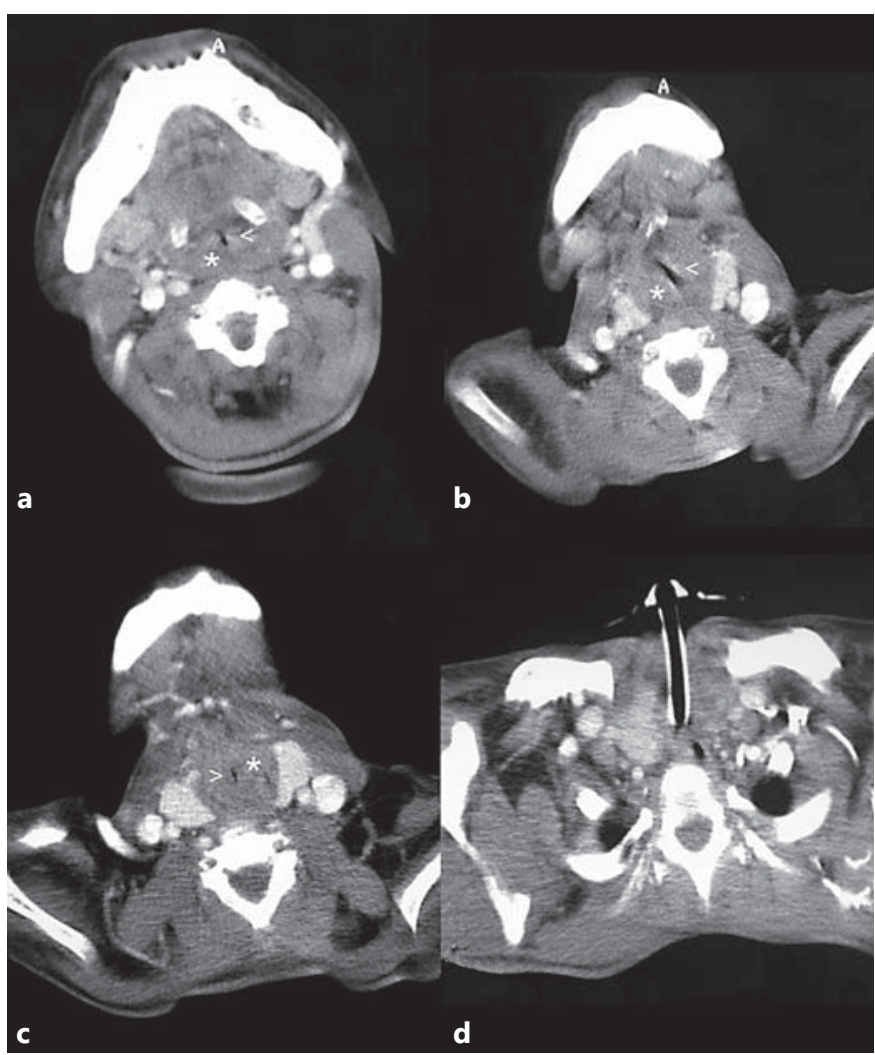

Fig. 2. Neck computed tomography showing an increased soft tissue thickening (asterisk) of the oropharynx (a) and the larynx (b, c) with a striking decrease of the aerial lumen (arrowhead) and the realization of a tracheostomy $(\mathbf{d})$.

\section{Case Report}

A 19-year-old, male patient with the diagnosis of MPS II syndrome by the determination of urinary GAGs (70 mg/24-h urine, predominantly dermatan sulfate but also heparan sulfate), plasma iduronate-2-sulfatase enzymatic activity $(216 \mathrm{pmol} / \mathrm{h}$ per $\mathrm{ml}$ (control of 2,068 $\mathrm{pmol} / \mathrm{h}$ per $\mathrm{ml}$ )) and the G374sp mutation at the nucleotide c.1246 of the gene encoding for the iduronate-2-sulfatase, was admitted to the emergency department because of shortness of breath, increase in the respiratory secretions and orthopnea. The patient was afebrile and cardiopulmonary auscultation showed tachypnea with an inspiratory stridor which was accentuated in the decubitus position. The patient referred a history of mitroaortic valve disease, obstructive sleep apnea treated with continuous positive airway pressure, giant inguinal hernia operated in childhood, recurrent upper respiratory tract infections in infancy and mild mental retardation.

On examination, the patient was small and nonobese (height $130 \mathrm{~cm}$, weight $40 \mathrm{~kg}$ ) and had a rough phenotype with macrocephaly, prominent eyes, coarse nose, thick lips and eyebrows, broad hands with fat thumbs, short limbs, and a tendency to semiflexion of the fingers. The tongue was enlarged and the soft palate and uvula were thickened. The abdomen was globulous with a hepatomegaly of 4-finger width, and the distal joints showed stiffness and increased size.

The blood analysis, including serum glucose, serum creatinine, liver enzymes, and $\mathrm{N}$-terminal pro-B-type, natriuretic peptide levels, was within normal limits. The basal arterial blood gas showed a $\mathrm{pH}$ of 7.4, a $\mathrm{pO}_{2}$ of $67 \mathrm{~mm} \mathrm{Hg}$ and a $\mathrm{pCO}_{2}$ of $73 \mathrm{~mm} \mathrm{Hg}$. The electrocardiogram was in sinus rhythm, and the chest radiograph showed no cardiomegaly. The echocardiogram revealed a preserved and nonhypertrophic left ventricle with a severe mitral valve regurgitation in relation to subvalvular and valvular thickening, retraction and leaflet coaptation defect (fig. 1). Also, a mildly thickened aortic and tricuspid valve with mild tricuspid and moderate aortic regurgitation were seen. No pericardial effusion or thrombi were found.

Neck computed tomography showed a hypertrophy of the Waldeyer's ring with partial obliteration of the valleculae and both pyriform sinuses and a filiform oropharynx, larynx and trachea lumen (fig. 2). Ear computed tomography revealed occupation of the mastoid air cells compatible with mastoiditis and thickening of the middle ear wall compatible with chronic otitis media. The audiometry was consistent with bilateral mixed hearing loss, and the ophthalmologic study showed bilateral optic neuropathy with demyelinating involvement.

The patient was initially treated with noninvasive ventilation having only a partial response. For this reason, a formal tracheostomy was performed with gradual improvement of his respiratory symptoms and the basal arterial blood gases ( $\mathrm{pH}$ of 7.44, a $\mathrm{pO}_{2}$ of $93 \mathrm{~mm} \mathrm{Hg}$ and a $\mathrm{pCO}_{2}$ of $41 \mathrm{~mm} \mathrm{Hg}$ ). Despite this, the patient died a few months later due to progressive obstructive airway disease.

His brother, 6 years younger, also has a MPS II syndrome with mild retardation, cord compression secondary to narrowing of the spinal canal at the bulbo-medullary level, mild aortic valve regurgitation, and obstructive airway disease under continuous positive airway pressure treatment. The genetic study of the younger brother also evidenced the same G374sp mutation, and the patient, who 
is now 17 years old, is under treatment with weekly intravenous infusions of recombinant iduronate-2-sulfatase (Elapraser (Shire Human Genetic Therapies, Inc., Cambridge, Mass., USA)). Also, a genetic analysis was carried out in their mother that confirmed the same G374sp mutation as her 2 sons.

\section{Discussion}

MPS II syndrome is an X-linked, recessive disorder that usually shows a vertical transmission. The most usual clinical manifestations are short stature, coarse facial features, mental retardation, respiratory and cardiovascular complications, bowel dysfunction, organomegaly, umbilical or inguinal hernias, hearing problems, ocular impairment, and joint contractures. However, the most common causes of death, which usually occurs within the second decade of life, are the obstructive airway disease and the heart failure due to valvular dysfunction [Schwartz et al., 2007; Jones et al., 2009; Kampmann et al., 2011].

Ear, nose, throat, and respiratory problems are very common in patients with MPS and are often among the first symptoms to appear, tending to progress with age. Clinical manifestations include dyspnea, difficulties with clearing secretions, cough, wheezing, and recurrent bronchitis or pneumonia. The GAGs deposition may lead to gingival hyperplasia, mucosal edema, distension of the tongue, adenoids and tonsils, and mucoid secretions, favoring chronic rhinosinusitis, chronic otitis media, upper airway obstruction, obstructive sleep apnea, and impaired exercise tolerance [Berger et al., 2012]. In fact, progressive deposition of GAGs in the soft tissue of the throat and trachea is thought to be responsible for the airway dysfunction and obstruction, which characterize the syndrome. Equally, other physical characteristics, which include abnormalities in the shape and structure of the ribs, abdominal organ enlargement, short neck, flattened nasal bridge, high epiglottis, and immobile jaw, may further contribute to these respiratory problems [Kamin, 2008]. Also, some alveolar and interstitial pulmonary involvement by GAGs deposition may favor restrictive pulmonary disease [Semenza and Pyeritz, 1988].

Moreover, progressive cardiac valve pathology is the most prominent and uniform cardiac manifestation (6090\%) [Fesslová et al., 2009]. The GAGs accumulation affects the subvalvular and valvular structures producing a distal thickening of the leaflets, loss of mobility and valve regurgitation, especially of the mitral and aortic valves. Valvular dysfunction usually emerges silently, contributing in the long term to left ventricular hypertrophy, systolic and diastolic dysfunction, cardiac arrhythmias, congestive heart failure, and early mortality. Also, GAGs accumulation may favor conduction abnormalities, ventricular hypertrophy and diffuse intimal proliferation with narrowing of the coronary arteries and the thoracic and the abdominal aorta [Taylor et al., 1991; Leal et al., 2010; Braunlin et al., 2011; Kampmann et al., 2011].

Many parameters have been used to evaluate the severity and to monitor the progression and the response to therapy in this syndrome. Among them, we find the urinary GAG determination, which shows those patients with higher urinary GAGs levels to have a worse prognosis, the measurement of the height and head circumference or the determination of bone deformities, mobility, self-care, social functioning, or quality of life [Beck et al., 2010]. Similarly, attempts have been made to link the phenotype with the genetic studies.

The IDS gene, located on chromosome Xq28, consists of 9 exons encoding a 550-amino-acid IDS protein. To date, more than 350 mutations have been identified in the IDS gene with the exonic point mutations G374sp, comprising half of the mutations, followed sequentially by small deletions, altered splicing, gross deletions, small insertions, complex rearrangements, small indels, and gross insertions/duplications. In a study of 155 unrelated individuals with MPS II, Froissart et al. [2007] identified 27 large and 128 small IDS gene alterations of which 96 were different. Meanwhile, Zhang et al. [2011] found in 38 Chinese patients with Hunter syndrome that point mutations constituted the major form, while mutations in codon p.R468 defined the largest number of patients in their cohort. Otherwise, Gort et al. [1998] revealed in a study that included 31 Spanish families with MPS II that the most frequent mutation, seen in $10 \%$ of the cases, was the G374sp and that this mutation was related with a milder phenotype. In a molecular analysis of 29 out of 41 Italian patients with Hunter syndrome, Filocamo et al. [2001] reported missense mutations, nonsense mutations, new mutations, small deletions or splice mutations, and major structural alterations such as large deletion and rearrangements in $55.2,20.7,17.2,13.8$ and $10.3 \%$ of the cases, respectively.

This extensive gene variability may be explained by the presence of several transcripts which favors a complex system of intron splicing that makes the IDS gene susceptible to splicing mutations. This is compounded by a pseudogene, located $80 \mathrm{~kb}$ downstream of the transcribed IDS, that seems to be responsible for various of the IDS gene mutations and gene rearrangements [Froissart et al., 2007; Zhang et al., 2011]. These data further confirm the 
extreme heterogeneity of IDS gene alterations and explain the high degree of the clinical presentations. Although to date, some progress has been made in establishing the genotype-phenotype correlations in order to provide the likely prognosis and the response to therapy, it still remains difficult, even more when some patients carrying the same alteration may present with different phenotypes, suggesting that factors other than the IDS gene (e.g. other genes, environmental factors) may modulate the clinical phenotype [Froissart et al., 2007].

Although it has been demonstrated that enzyme replacement therapy with idursulfase is effective in relation to functional capacity (distance walked in $6 \mathrm{~min}$ and forced vital capacity), liver and spleen volumes and urine glycosaminoglycan excretion in patients with MPS II compared with placebo, there is no available evidence on outcomes such as improvement in growth, sleep apnea, cardiac function, quality of life and mortality [da Silva et al., 2011; Muenzer et al., 2011]. Similarly, though stabili- zation of valvular disease in selected patients over a limited study period has been observed, enzyme replacement therapy does not appear to prevent progression of leftsided cardiac valve thickening and dysfunction if the enzyme replacement begins in childhood or adulthood [Okuyama et al., 2010]. By contrast, if initiated in the first year of life and onwards, patients appear phenotypically normal with no echocardiographic abnormalities either before, or after, enzyme replacement treatment [Braunlin et al., 2011].

As we can see, the MPS II syndrome entails a broad range of symptoms with different grades of severity. Two of the most significant areas of variability concern the degree of mental retardation and survival. Although the term mild phenotype, as seen in our 2 MPS II patients, is used by physicians in comparing the absence or presence of mental retardation, the effects of even mild disease involve a high morbidity and mortality, especially between the third and fourth decade of life.

\section{References}

Beck M: Mucopolysaccharidosis type II (Hunter syndrome): clinical picture and treatment. Curr Pharm Biotechnol 12:861-866 (2011).

Beck M, Muenzer J, Scarpa M: Evaluation of disease severity in mucopolysaccharidoses. J Pediatr Rehabil Med 3:39-46 (2010).

- Berger KI, Fagondes SC, Giugliani R, Hardy KA, Lee KS, et al: Respiratory and sleep disorders in mucopolysaccharidosis. J Inherit Metab Dis 2012, E-pub ahead of print.

- Braunlin EA, Harmatz PR, Scarpa M, Furlanetto B, Kampmann C, et al: Cardiac disease in patients with mucopolysaccharidosis: presentation, diagnosis and management. J Inherit Metab Dis 34:1183-1197 (2011).

da Silva EM, Strufaldi MW, Andriolo RB, Silva LA: Enzyme replacement therapy with idursulfase for mucopolysaccharidosis type II (Hunter syndrome). Cochrane Database Syst Rev 11:CD008185 (2011).

-Fesslová V, Corti P, Sersale G, Rovelli A, Russo P, et al: The natural course and the impact of therapies of cardiac involvement in the mucopolysaccharidoses. Cardiol Young 19:170178 (2009)

Filocamo M, Bonuccelli G, Corsolini F, Mazzotti R, Cusano R, Gatti R: Molecular analysis of 40 Italian patients with mucopolysaccharidosis type II: new mutations in the iduronate-2-sulfatase (IDS) gene. Hum Mutat 18:164-165 (2001).
Froissart R, Da Silva IM, Maire I: Mucopolysaccharidosis type II: an update on mutation spectrum. Acta Paediatr Suppl 96:71-77 (2007).

Gort L, Chabás A, Coll MJ: Hunter disease in the Spanish population: molecular analysis in 31 families. J Inherit Metab Dis 21:655-661 (1998).

-Jones SA, Almássy Z, Beck M, Burt K, Clarke JT, et al: Mortality and cause of death in mucopolysaccharidosis type II - a historical review based on data from the Hunter Outcome Survey (HOS). J Inherit Metab Dis 32:534-543 (2009).

Kamin W: Diagnosis and management of respiratory involvement in Hunter syndrome. Acta Paediatr Suppl 97:57-60 (2008).

Kampmann C, Beck M, Morin I, Loehr JP: Prevalence and characterization of cardiac involvement in Hunter syndrome. J Pediatr 159:327331 (2011).

Leal GN, de Paula AC, Leone C, Kim CA: Echocardiographic study of paediatric patients with mucopolysaccharidosis. Cardiol Young 20:254-261 (2010).
Muenzer J, Beck M, Eng CM, Giugliani R, Harmatz P, et al: Long-term, open-labeled extension study of idursulfase in the treatment of Hunter syndrome. Genet Med 13:95-101 (2011).

Okuyama T, Tanaka A, Suzuki Y, Ida H, Tanaka T, Cox GF, et al: Japan Elaprase Treatment (JET) study: idursulfase enzyme replacement therapy in adult patients with attenuated Hunter syndrome (Mucopolysaccharidosis II, MPS II). Mol Genet Metab 99:18-25 (2010).

-Schwartz IV, Ribeiro MG, Mota JG, Toralles MB, Correia $\mathrm{P}$, et al: A clinical study of 77 patients with mucopolysaccharidosis type II. Acta Paediatr Suppl 96:63-70 (2007).

-Taylor DB, Blaser SI, Burrows PE, Stringer DA, Clarke JT, Thorner P: Arteriopathy and coarctation of the abdominal aorta in children with mucopolysaccharidosis: imaging findings. AJR Am J Roentgenol 157:819-823 (1991).

Young ID, Harper PS, Newcombe RG, Archer IM, Newcombe RG: A clinical and genetic study of Hunter's syndrome 2. Differences between the mild and severe forms. J Med Genet 19: 408-411 (1982).

Zhang H, Li J, Zhang X, Wang Y, Qiu W, et al: Analysis of the IDS gene in 38 patients with Hunter syndrome: the c.879G $>A$ (p.Gln293Gln) synonymous variation in a female create exonic splicing. PLoS One 6:e22951 (2011) 